\title{
Alpha I antitrypsin deficiency: a rare allele in patients from south of Italy
}

\begin{abstract}
Congenital $\alpha 1$-antitrypsin deficiency (AATD) is an autosomal recessive disorder, in Italy it is estimated that 1 in 5000 individuals may suffer from severe AATD The AATD pathogenesis is directly related to gene mutations, which are highly polymorphic: in fact, more than 120 genetic variants closely associated with specific plasma glycoprotein concentrations have been identified. All the variants have a different clinical significance as they can cause an increase of occurrence of some pathologies such as emphysema, acute or chronic liver disease, cirrhosis, or liver failure. In particular, emphysema affects $54 \%$ of patients diagnosed with this deficit. The purpose of our study was to perform a mutational analysis of the AAT gene in order to highlight a genotype-serum correlation of AAT: we found subjects heterozygous for the rare allele PiMProcida and correlated its presence with a marked lowering of serum AAT levels.
\end{abstract}

Keywords: alpha1 antitrypsin deficit, emphysema, SERPINA1
Volume 6 Issue 2 - 2019

\section{Maddaloni V, Pepe N, Morano F, Lanzo M, D’Arco D, Atripaldi L}

Molecular genomics lab, Chemical biochemistry Unit, Monaldi Hospital, Italy

\section{Correspondence: Maddaloni $V$, Molecular genomics lab, Chemical biochemistry Unit, AORN deiColli, Monaldi Hospital, V.L. Bianchi, 80 I3I Naples, Italy, Tel +393356220323,} mail valeria.maddalon@gmail.com

Pepe N, Molecular genomics lab, Chemical biochemistry Unit, AORN deiColli, Monaldi Hospital,V.L. Bianchi, 80I3I Naples, Italy,

Email nicola.pepe@fastwebnet.it

Received: January 28, 2019 | Published: March 29, 2019

\section{Introduction}

Congenital $\alpha 1$-antitrypsin deficiency (AATD) is a hereditary genetic disorder transmitted through an autosomal recessive character with various forms of penetration and expressiveness. ${ }^{1}$

This condition is characterized by reduced or absent levels of $\alpha 1$-antitrypsin that do not derive from a lack of protein synthesis, but depend on a misfolding of the latter which leads to incorrect polymerization. ${ }^{2}$ In Europe, it is believed that the proportion of people affected by AAT deficiency is from 1 in 1,600 to 1 in 2,000, for a total amount of about 125,000 people, but the prevalence of this disorder varies from population to population: the highest concentrations are found in North-European populations and in those deriving from them. These concentrations tend to decrease according to a NorthSouth gradient..$^{3-5}$

In Italy it is estimated that 1 in 5000 individuals may suffer from severe AATD, with frequency peaks in some areas of 1 case per 2000 inhabitants. Several epidemiological studies have shown that this disease is a misdiagnosed condition: in fact, despite the considerable effort to characterize the affected subjects and despite being one of the most common and serious inherited disorders worldwide, only a portion of subjects affected by this deficit currently has been recognized.

\section{Alpha I antitrypsin}

Alpha 1 antitrypsin (AAT) is a water-soluble and tissue-diffusible, medium sized $(6.7 \times 3.2 \mathrm{~nm})$ circulating glycoprotein consisting of 394 amino acids, with a weight of 52 kiloDalton $(\mathrm{kDa})$ and a half-life of 4-5 days. ${ }^{6-8}$

Over $80 \%$ of AAT is synthesized and secreted by hepatocytes, but additional quantities are also produced by monocytes, macrophages, pancreas, lung alveolar cells, enterocytes, endothelium and some cancers. The human organism produces $34 \mathrm{mg} / \mathrm{kg}$ per day of AAT, with consequent plasma concentrations of 1-3 g/L. From plasma, $80 \%$ of it spreads to interstitial tissues and another $0.5-10 \%$ reaches biological fluids. ${ }^{9-13}$
Discovered for the first time in 1955, it is considered the Serpin superfamily archetype since it was the first responsible agent for blood's antiprotease activity. ${ }^{14-16}$

As a Serpin family member, the AAT shares a common structure consisting of $3 \beta$-sheets (ABC), $9 \alpha$-helices and a mobile reactive loop composed by 20 amino acids, within which there is an active site (Met358-Ser359) which acts as a pseudosubstrate for the target protease. ${ }^{4,17,18}$ This structure undergoes an important conformational change when it comes into contact with the protease. ${ }^{5,19-20}$ This not only allows inhibition of the protease activity, but also preparation for the destruction of the target molecule. ${ }^{21-23}$ This whole process consists of an inhibiting mechanism called "substrate suicide" characterized by the irreversible serpin-protease complex formation, which is internalized and subsequently degraded. The AAT, also known as Protease Inhibitors $(\alpha 1-\mathrm{PI})$ and/or SERPINA1 (serine protease inhibitor, group A, member 1), functions to inhibit a series of serine proteases, such as pancreatic trypsin, cathepsin $\mathrm{G}$ and protease3, but its main target is Neutrophilic Elastase (NE), which is released at the pulmonary level after neutrophil activation. ${ }^{9-14}$ The structure of the AAT also represents the "Achilles' heel" molecule itself because point mutations in certain regions make the protein vulnerable to aberrant conformational transition, causing the function impairment. ${ }^{17}$

The AATD pathogenesis is directly related to gene mutations, which are highly polymorphic: in fact, more than 120 genetic variants closely associated with specific plasma glycoprotein concentrations have been identified. ${ }^{17-24}$ Each variant name contains a letter that describes migration velocity in an electrofocusing test, the assay used in phenotyping: S-variants are slow, M-variants have a medium velocity and, also, F-variants are fast. Besides the autosomal recessive inheritance, we also refer to codominance because two different alleles of the same gene can be active and influence the phenotypic expression; this expression causes the genotype to be defined - indeed - composite heterozygote. ${ }^{25}$

All the variants have a different clinical significance as they can cause an increase of occurrence of some pathologies such as emphysema, acute or chronic liver disease, cirrhosis, or liver failure. 
In particular, emphysema affects $54 \%$ of patients diagnosed with this deficit. ${ }^{3}$ The purpose of our study was to perform a mutational analysis of the AAT gene in order to highlight a genotype-serum correlation of AAT.

\section{Materials and methods}

This study focuses on the most common mutated variants on SERPINA1 gene, $\mathrm{Pi}^{* \mathrm{~S}}$ and $\mathrm{Pi} * \mathrm{Z}$ and on the rarer $\mathrm{PiM}_{\text {Malton }}$ (rs775982338) and PiMProcida (rs28931569) variants detection. We have surveyed 72 patients belonging to the AORN Monaldi, Ospedali dei Colli - Naples, Italy. Three different kits were used in order to identify the mutations present in the SERPINA1 gene, in a sample of human gDNA extracted from peripheral blood in EDTA. For the detection of $\mathrm{Pi}^{*} \mathrm{~S}$ and $\mathrm{Pi} * \mathrm{Z}$ variants we used the AAt $\mathrm{Pi} \mathrm{Z}^{*} \mathrm{\textrm {Pi }}{ }^{*} \mathrm{~S}$ LightMix ${ }^{\circledR}$ from TIB MOLBIOL; for the two rare variants PiM $_{\text {Malton }}$ and PiMProcida we used probes produced by TIB MOLBIOL at our request.

For each blood sample we have obtained informed consent. gDNA extraction was performed with automatic instrumentation, using the MagNA Pure Compact Instrument and the related MagNA Pure Compact Nucleic Acid Isolation Kit I, produced by Roche Diagnostics; subsequently we determined the right concentration and then we evaluated DNA's extracted purity through the NanoDrop 2000, by ThermoScientific. Real Time PCR experiments were made following manufacturer instructions with the ROCHE Cobas z 480.

\section{Results}

Of the 72 patients we analyzed, 10 are heterozygous $\mathrm{Pi}$ *S (allele frequency 0.21 ), 4 heterozygous $\mathrm{Pi}^{*} \mathrm{Z}$ (allelic frequency 0.09 ) and 5 heterozygous PiMProcida (allelic frequency 0.11); one patient turned out to be composite heterozygous Pi*Z/PiMProcida. (Figure 1) The most frequent allele is $\mathrm{Pi}$ * $\mathrm{S}$, with a percentage of 21,28 , while $\mathrm{Pi}^{*} \mathrm{Z}$ frequency rate is $7.27 \%$ and $9.09 \%$ for PiMProcida.

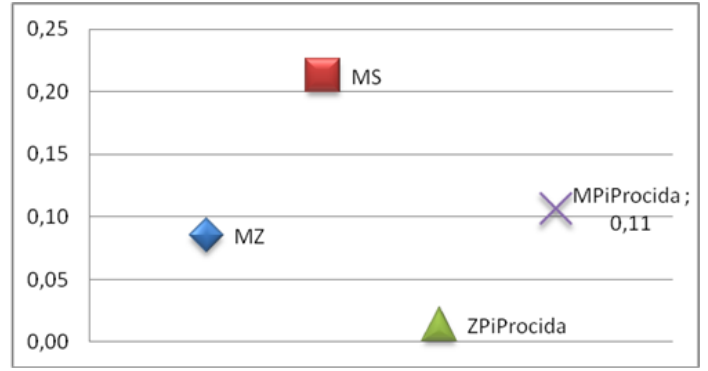

Figure I Allele frequencies in our cohort.

The family of the probands has not always been subjected to molecular screening and among all the subjects analyzed only 17 of them are familiar. Subjects with the $\mathrm{Pi}^{*} \mathrm{~S}$ allele had a plasma concentration of AAT between 1.20 and $1.24 \mathrm{~g} / \mathrm{L}$; those with the $\mathrm{Pi}$ * Z allele had a concentration between 0.79 and $0.97 \mathrm{~g} / \mathrm{L}$; the PiMProcida involves a more marked lowering of circulating AAT with respect to the $\mathrm{Pi}^{*} \mathrm{~S}$ allele, the concentration of these subjects varies between 0.70 and $0.80 \mathrm{~g} / \mathrm{L}$. It is interesting to note the dramatic lowering of AAT in serum of the compound heterozygous subject who has a plasma concentration of $0.24 \mathrm{~g} / \mathrm{L}$.

In addition, a subject in our cohort had a severe protein deficiency, whose concentration of plasma AAT was $0.74 \mathrm{~g} / \mathrm{L}$, but when subjected to a first analysis for the $\mathrm{Pi}{ }^{*} \mathrm{~S}$ and $\mathrm{Pi}$ Z Z variants was wild type for both alleles. Subsequently, it was re-evaluated for the two rarer variants when implemented, resulting heterozygous for the Procida variant. (Figure 2)

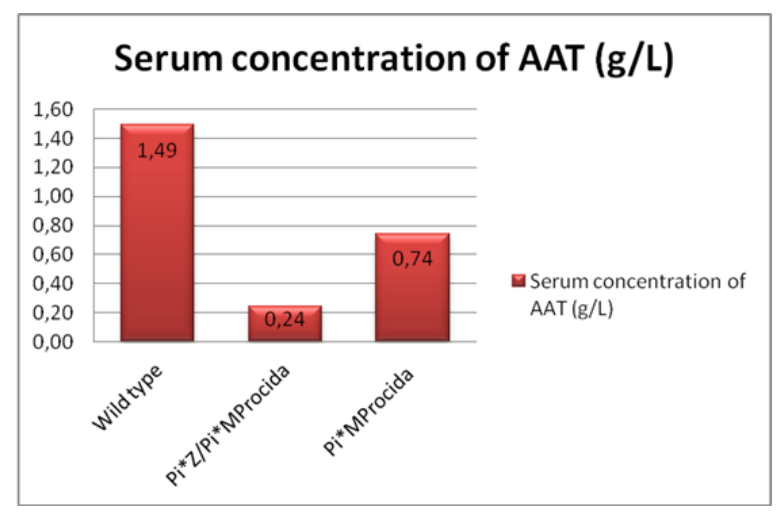

Figure 2 Dramatic lowering of serum AAT levels in patients heterozygous for the Procida variant $\left(\mathrm{PiM}_{\text {Procida }}\right)$.

\section{Discussion and conclusions procida}

The Null variants class of the SERPINA1 gene is characterized by the following variants:

$\begin{array}{ll}\text { - } & \text { Bellingham } \\ \text { - } & \text { Granite Falls } \\ \text { - } & \text { Bolton } \\ \text { - } & \text { Hong Kong } \\ \text { - } & \text { Mattawa } \\ \text { - } & \text { Procida }^{27}\end{array}$

All these variants are known as "Null" because they insert premature stop codons. Their presence in the Bellingham and Granite Falls variants may involve the inhibition of the A1-Antitrypsin protein's synthesis, while in the Hong Kong and Mattawa variants the A1AT protein's synthesis undergoes intracellular degradation. The Procida variant, on the other hand, is another rare and unique variant of the SERPINA1's gene which originates from a medium-sized deletion; this deletion involves the removal of a coding part of SERPINA1's gene, namely the part that-under natural circumstances-enables the A1AT protein's synthesis. ${ }^{26}$

The Procida variant distinguishes itself from the normal variant of the A1AT's gene by its loss of 4 coding exons (from exon 2 to exon 5 ) and by the presence of the Ia, Ib and Ic non-coding exons. Usually greater deletions have a strong impact on phenotype: they lead tothe occurrence of genetic diseases such as Duchenne Muscolar Dystrophy, beta thalassaemia or heamophilia type A. The present case's deletion involves the lack of synthesis of a mRNA strand, which then results in the inhibition of the A1AT protein's synthesis. ${ }^{26}$ It was first detected in the late period of the 80's, in an Italian American family, by Takahashi et $\mathrm{al}^{26}$ the researchers subjected the family to a genetic investigation and the Index Case turned out to be a 40 year old male patient, who was suffering from emphysema and that presented with both the Procida variant and the (heterozygous) Null allele. In order to codify the specific region they used the PuvII endonuclease, which produced a $2.9 \mathrm{~kb}$ fragment instead of the normal fragments $(2.7$ and $0.2 \mathrm{~kb}) .^{27}$

Since this variant is fairly homogeneous in the Italian peninsula it's likely that this variant originated from Italy. It belongs to the M-like group, namely the group characterized by mutations with a regular phenotypic aspect; in fact, these mutations do not cause the alteration of the protein's isoelectric point, and in the isoelectrophoretic analysis, as a result, they behave just like the normal $\mathrm{M}$ group variants (that is the reason why they can be mistaken). But the sequencing of the gene 
allows their identification: they are caused by a point mutation $(\mathrm{T} \rightarrow \mathrm{C})$ in exon 2 of the gene, on codon 41, which leads to an amino acid mutation (Leu41CTG $\rightarrow$ Pro41CCG). ${ }^{28}$

Takahashi's study has not detected further changes to the sequence, so it is possible to say that this simple amino acid substitution leads to a structural breakdown or a weakening of AAT protein's alpha-helix A. Furthermore, the analysis of the Index Case's karyotype showed that the cells were totally normal (46 XY), making clear that the loss of the A1AT's coding exons did not depend on a chromosomal deletion. ${ }^{26}$

Since prolin does not support $\alpha$-helix, this mutation modifies the tertiary structure of the A1AT molecule. Given this, and knowing that the in vivo half-life of MProcida is similar to the normal M1 molecule (Val213), the most probable explanation for the profound deficiency status associated with MProcida is that the molecule could be degraded intracellularly before secretion. ${ }^{27}$

In fact, the $\mathrm{M}_{\text {Procida }}$ mutation is responsible for lower plasma and pulmonary concentrations of AAT (about $80 \%$ of the produced protein

Table I It shows the Null variants do entail on a pathological basis

\begin{tabular}{lllll}
\hline $\begin{array}{l}\text { Null variants of the } \\
\text { SERPINA1 gene }\end{array}$ & $\begin{array}{l}\text { Exons } \\
\text { involved }\end{array}$ & $\begin{array}{l}\text { Production of a stablem- } \\
\text { RNA strand }\end{array}$ & Mutation & $\begin{array}{l}\text { A1-antitrypsin } \\
\text { Synthesis (A1AT) }\end{array}$ \\
\hline Bellingham & III & No & & Synthesis blocked \\
Granite Falls & II & No & Small substitution, small & - \\
Bolton & V & - & insertion, small deletion. & Intracellular aggregation \\
Hong Kong & IV & Maybe & & $\begin{array}{l}\text { Intracellular degradation } \\
\text { of A1AT }\end{array}$ \\
Mattawa & V & Yes & & Big deletion \\
Procida & II-III-IV-V & No & & Synthesis blocked \\
\hline
\end{tabular}

Unlike previous research, through this prospective study carried out in the Molecular Genomics sector of Azienda Ospedaliera Specialistica dei Colli (Monaldi - Cotugno - C.T.O.) we managed to analyze a large cohort of patients, making it possible to highlight a fairly high frequency of PiMProcida of the SERPINAlgene: in fact, despite being a rather rare variant it clearly shows a higher incidence in our regional territory than anywhere else.

Already from this first part of the work, it has emerged how the variant Procida can have an important impact on the phenotype: we have indeed found that it involves a strong lowering of serum levels of A1AT even when not accompanied by the more frequent variations $\mathrm{S}$ and $\mathrm{Z}$. We could observe in fact, its presence in heterozygosity lowers serum levels by $50 \%$ compared to the wild type, whilst its contribution to the phenotype becomes more important when in association with the more common variant $Z$. In the next year we plan to implement the sequencing of the entire SERPINA1 gene; certainly the population that we will examine will be expanded and we will review all the previous cases to evaluate the presence of rare variants not diagnosed in this first screening.

\section{Acknowledgments}

None.

\section{Conflicts of interest}

Authors declare that there is no conflict of interest. is immediately degraded) and for the related lung problems that may occur, but it is not kept inside the hepatocytes. Therefore, from a clinical point of view, patients with this mutation are not at risk of liver diseases, unlike what happens in $\mathrm{M}_{\text {Malton }}$ patients. ${ }^{28,29}$

Besides, Toshihiro Nukiwa's study has shown how the point mutation causes a slight decrease in the $\mathrm{M}_{\text {Procida }}$ 's association constant with neutrophil elastase (normal $9.3 \pm 0.8 \times 106 \mathrm{M}-1 \mathrm{~S}-1, \mathrm{M}_{\text {Procida }}$ $\left.7.0 \pm 0.1 \times 10^{6}\right)$. Hence, together with the profound deficiency status associated with $\mathrm{M}_{\text {Procida }}$, the mutation also makes the molecule relatively useless in providing a protective shield against anti-neutrophil elastase for the lung. ${ }^{25}$ According to the study, the clinical importance of the Null alleles becomes evident when inherited with another Null allele or with a deficient allele such as $\mathrm{Z}$ or "S". In such circumstances, as in the Index Case's one, serum levels of A1AT are low enough to put the affected patient at high risk of developing emphysema. ${ }^{24}$ The Procida variant has never been fully described in any study: as a very rare variant, it has never been found in a large cohort of patients. This is why further studies are needed to understand what further allelic combinations of Null variants do entail on a pathological basis (Table $1)$.

\section{References}

1. Luisetti M, Seersholm N. Alpha1-antitrypsin deficiency. 1: epidemiology of alpha1-antitrypsin deficiency. Thorax. 2004 ;59(2):164-169.

2. Didier Belorgey, Peter Hägglöf, Karlsson Li S, et al. Protein Misfolding and the Serpinopathies. Prion. 2007;1(1):15-20.

3. Ilaria Ferrarotti, Marina Gorrini, Roberta Scabini, et al. Diagnosi di laboratorio del deficit ereditario di $\alpha 1$-antitripsina. Biochimica clinica. 2009;33(1):6.

4. Bruno Balbi, Laura Pini, Luciano Corda, et al. Trattato italiano di pneumologia-Enfisema da deficit di AAT. 2004;3:1943-1963.

5. David G Parr, Beatriz Lara. Clinical utility of alpha-1 proteinase inhibitor in the management of adult patients with severe alpha-1 antitrypsin deficiency: a review of the current literature. Drug Des Devel Ther. 2017;11:2149-2162.

6. Irving JA, Steenbakkers PJ, Lesk AM, et al. Serpins in prokaryotes. Mol Biol Evol. 2002;19(11):1881-1890.

7. Patston PA. Serpins and other serine protease inhibitors. Immunol Today. 2000;21:354.

8. Ragg H, Lokot T, Kamp PB, et al. Vertebrate serpins: construction of a conflict-free phylogeny by combining exon-intron and diagnostic site analyses. Mol Biol Evol. 2001;18(4):577-584.

9. F de Serres, I Blanco. Role of alpha 1 antitrypsin in human health and disease. J Intern Med. 2014;276(4):311-315. 
10. American Thoracic Society, European Respiratory Society. American Thoracic Society/European Respiratory Society statement: standards for the diagnosis and management of individuals with alpha1-antitrypsin deficiency. Am J Respir Crit Care Med. 2003;168(7):818-900.

11. Stoller JK, Aboussouan LS. Alpha1-antitrypsin deficiency. Lancet. 2005;365(9478):2225-2236

12. Stoller JK, Aboussouan LS. A review of a1-antitrypsin deficiency. Am J Respir Crit Care Med. 2012;185(3):246-259.

13. Janciauskiene SM, Bals R, Koczulla R, et al. The discovery of $\alpha 1$-antitrypsin and its role in health and disease. Respir Med. 2011;105(8):1129-39.

14. Schultze HE, Goilner I, Heide K, et al. Alpha-globulin desmenschli chennirmal serums. Z Naturforsch. 1995;10:463.

15. Carrell RW, Pemberton PA, Boswell DR. The serpins: evolution and adaptation in a family of protease inhibitors. Old Spring Harb Symp Quant Biol. 1987;52:527-535.

16. Gettins PG. Serpin structure, mechanism, and function. Chem Rev. 2002;102(12):4751-4804.

17. Lomas DA, Parfrey H. $\alpha 1$-Antitrypsin deficiency4: Molecular pathophysiology. Thorax. 2004;59(6):529-535.

18. Huntington JA, Read RJ, Carrell RW. Structure of a serpinprotease complex shows inhibition by deformation. Nature. 2000;407(6806):923-926.

19. Patrizia Lombari. Modulazione dell'espressione della serpina Proteasi Nexina 1 (PN1) in sistemi cellulari neurali e gliali. 2005. 96 p.

20. Janciauskiene S. Conformational properties of serine proteinase inhibitors (serpins) confer multiple pathophysiological roles. Biochim Biophys Acta. 2001;1535(3):221-235.
21. Giorgio Bartolozzi. Alfa1-antitripsina-deficienza: un'alterazione conformazionale simile a quella dei prioni nella malattia di CreutzfeldJacob variante. Medico e Bambino pagine elettroniche. 2002;5(3):10.

22. Carrell RW, Lomas DA. Alpha1-antitrypsin deficiency-a model for conformational diseases. N Engl J Med. 2002;346(1):45-53.

23. Survival and FEV1 Decline in Individuals with Severe Deficiency of a1-Antitrypsin. Am J Respir Crit Care Med. 1998;158(1):49-59.

24. Progetto condotto dall'AORN, Azienda Ospedaliera dei Colli, MonaldiCotugno-CTO. Valutazione della presenza di mutazioni rare dell'Alfa 1 antitripsina in pazienti con patologie polmonari o epatiche idiopatiche non allele S o Z dipendenti, Napoli. 2017.

25. Genetic Home Reference. Alpha-1 antitrypsin deficiency. Lister Hill National Center for Biomedical Communications, U.S. National Library of Medicine, National Institutes of Health, Department of Health\& Human Services. 2018

26. Takahashi H, Crystalal RG. Alpha 1-antitrypsin Null (isola di procida): an alpha1-Antitrypsin Deficiency Allele Caused by Deletion of All alAntitrypsin Coding Exons. Am J Hum Genet. 1990;47(3):403-413.

27. Toshihiro Nukiwa, Ken Satoh, Fumitaka Ogushi, et al. Crystal. Characterization of the Gene and Protein of the al-Antitrypsin "Deficiency" Allele M . The journal of biological chemistry. 1988;263(30):15528-15534.

28. Giorgia Sala, Ilaria Ferrarotti, Maurizio Luisetti. Aspetti clinici e prognostici della patologia epatica o polmonare conseguente a deficit di alfa1-antitripsina: analisi sul Registro Italiano. Bollettino della Società Medico Chirurgica di Pavia. 2013;126(2):385-393.

29. Clinica delle Malattie dell'Apparato Respiratorio, Università degli Studi di Pavia, Fondazione IRCCS Policlinico San Matteo, Pavia, Italia. Bollettino della Società Medico Chirurgica di Pavia. 2015;126(2):385-39. 